\title{
Complicações Cardíacas em Longo Prazo da Síndrome do PRKAG2
}

\section{Long-Term Cardiac Complications of PRKAG2 Syndrome}

\author{
Luiz Pereira de Magalhães, ${ }^{1 \oplus}$ Eduardo Faria Soares de Magalhães, ${ }^{2}{ }^{\oplus}$ Jussara de Oliveira Pinheiro, ${ }^{1}$ Alex Teixeira \\ Guabiru, ${ }^{1}$ Francisco José Farias Borges dos Reis, ${ }^{1}$ Roque Aras $^{1}$ \\ Hospital Universitário Professor Edgard Santos, ${ }^{1}$ Salvador, BA - Brasil \\ Universidade Federal da Bahia - Faculdade de Medicina da Bahia, ${ }^{2}$ Salvador, BA - Brasil
}

\section{Introdução}

A síndrome do PRKAG2 é uma doença genética rara, de herança autossômica dominante, causada por mutações no gene que codifica a subunidade $\mathrm{Y}_{2}$ da proteína quinase ativada por AMP. A doença está associada ao acúmulo anormal de glicogênio nos cardiomiócitos, predispondo à hipertrofia ventricular, arritmias e morte súbita. ${ }^{1,2} \mathrm{~A}$ mutação foi primeiramente descrita por Gollob et al. em 2001, despertando, na comunidade médica, a importância do diagnóstico diferencial com miocardiopatia hipertrófica. ${ }^{3,4}$ Embora a prevalência da síndrome do PRKAG2 não esteja estabelecida, o número de casos pode estar aumentando devido à maior disponibilidade de genotipagem. No entanto, ainda não foram descritos possíveis fatores prognósticos na literatura. Considerando a possível gravidade do quadro clínico e a escassez de dados a respeito da história natural, o objetivo deste estudo foi avaliar a evolução clínica de pacientes portadores da síndrome do PRKAG2, analisando a incidência de complicações cardíacas em longo prazo.

\section{Métodos}

Trata-se de estudo observacional, ambispectivo, envolvendo membros de uma única família portadora de mutação Arg302Gln do gene PRKAG2. ${ }^{5}$ Foram coletados dados clínicos, e realizados eletrocardiograma (ECG), ecocardiograma, e estudo eletrofisiológico. Hipertrofia ventricular esquerda foi definida como espessura de septo interventricular ou de parede posterior $\geq 13 \mathrm{~mm}$ no ecocardiograma, sem causa aparente. O teste exato de Fisher foi utilizado para comparações entre variáveis categóricas, e o teste t de Student para amostras independentes foi usado para comparações entre médias das variáveis contínuas. Foram calculadas as razões de prevalência entre as variáveis de interesse e os desfechos clínicos. O desfecho primário foi implante de marcapasso (MP); o desfecho combinado foi definido pela

\section{Palavras-chave}

Flutter Atrial; Hipertrofia Ventricular Esquerda; Bloqueio Atrioventricular; Marca-Passo Artificial.

Correspondência: Luiz Pereira de Magalhães •

Hospital Universitário Professor Edgard Santos - Cardiologia - Clínica Médica Rua Dr. Augusto Viana, s/n. CEP 40110-060, Canela, Salvador, BA - Brasil E-mail: luizmagalhaes12@gmail.com

Artigo recebido em 25/01/2021, revisado em 21/05/2021, aceito em 28/07/2021

DOI: https://doi.org/10.36660/abc.20210062 ocorrência de implante de marca-passo (MP) ou morte súbita. O método de Kaplan-Meier foi utilizado para estimar a incidência cumulativa do desfecho combinado. Um valor de $p<0,05$ foi considerado estatisticamente significativo.

\section{Resultados}

Entre março de 1996 e janeiro de 2020, foram avaliados 16 indivíduos (63\% homens, $n=10)$ idade média de $40 \pm 11$ anos (Figura 1). As características basais dos pacientes estão descritas na Tabela 1. Três indivíduos (18\%) apresentaram morte súbita. Durante um tempo médio de acompanhamento de 15,1 $\pm 2,9$ anos, cinco (38\%) dos 13 pacientes remanescentes necessitaram de MP devido a bloqueio atrioventricular ou disfunção sinusal, em idade média de $44 \pm 6$ anos. O fenótipo predominante foi caracterizado por bradicardia sinusal e pré-excitação ventricular, encontrada em todos os pacientes, (Figura 2A); seis (46\%) tiveram flutter ou fibrilação atrial, e 7 (54\%) apresentaram hipertrofia ventricular esquerda ao ecocardiograma. Todos os pacientes apresentaram fração de ejeção preservada. Seis pacientes foram submetidos ao estudo eletrofisiológico, o qual foi consistente com a via fascículo-ventricular (Figura 2B). A medida dos intervalos basais mostrou intervalo HV curto e fixo (mediana=30 ms). Não houve indução de arritmia ventricular em nenhum paciente.

A incidência cumulativa do desfecho combinado (implante de MP e morte súbita) está apresentada na Figura 3. Os eventos cardiovasculares ocorreram antes dos 50 anos. A probabilidade de desenvolvimento do desfecho combinado aos 40 anos foi de 44\% (IC 95\%: 14-84\%). Também foram comparadas as características de pacientes submetidos a implante de MP e pacientes sem MP (Tabela 2). Flutter atrial foi significativamente mais prevalente nos pacientes que necessitaram de MP $(80 \%$ vs $13 \%, p=0,032)$, estando associada a probabilidade 6,4 vezes maior de evolução para esse desfecho.

\section{Discussão}

Analisamos a evolução clínica em longo prazo de portadores de mutação Arg302Gln do gene PRKAG2 em uma família. Foi observado o caráter precoce de acometimento cardiovascular nesses pacientes, com eventos significativos, como implante de MP ou morte súbita, ocorrendo antes dos 50 anos. Em relação ao eletrocardiograma, todos os pacientes apresentaram pré-excitação ventricular, frequentemente associado a bloqueio de ramo direito e bradicardia sinusal. De acordo com estudos prévios, a prevalência dessas anormalidades é variável e pode aumentar com a idade. ${ }^{6}$ 


\section{Carta Científica}

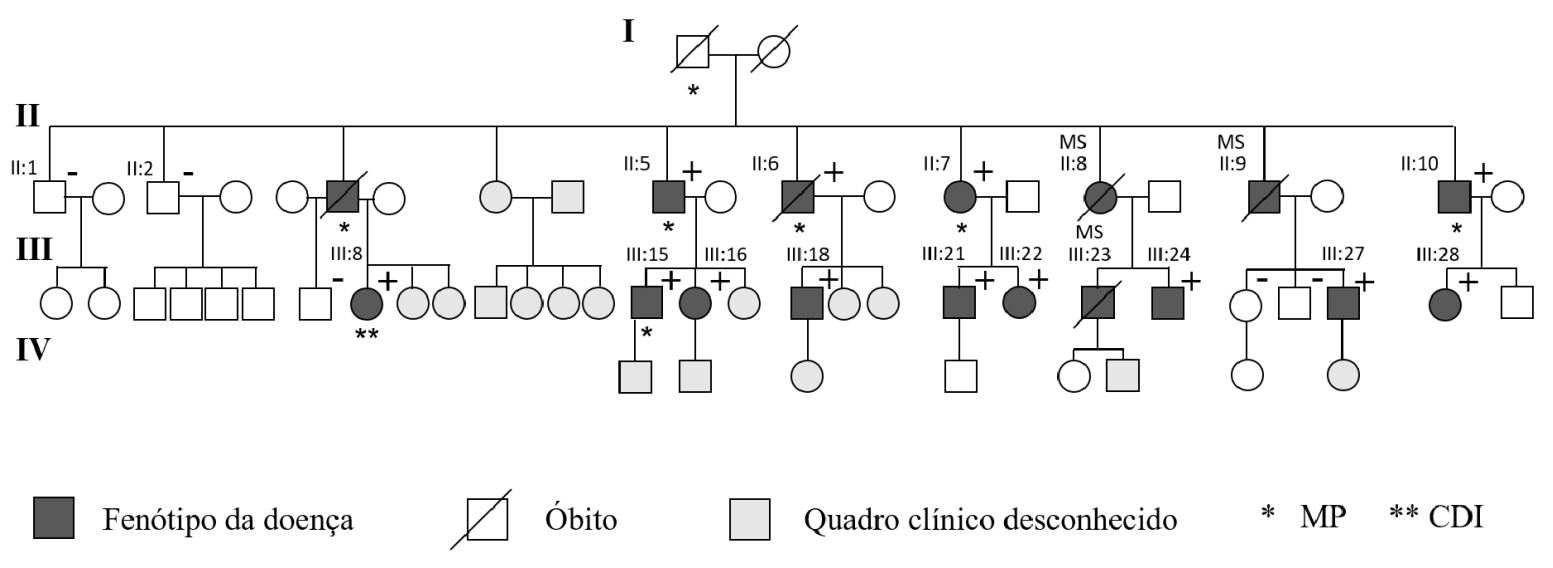

Figura 1 - Heredograma da familia. Individuos testados quanto à mutação do PRKAG2: (+) portadores; (-) não portadores da mutação. MP: marca-passo; CDI: cardioversor-desfibrilador implantável; MS: morte súbita.

Tabela 1 - Características clínicas dos pacientes ( $n=16)$ de uma única família portadora de mutação Arg302Gln do gene PRKAG2

\begin{tabular}{|c|c|c|c|c|c|c|}
\hline Paciente & Sexo & Idade & Morte Súbita & Arritmia SV & $\mathrm{DCl}$ & HVE \\
\hline II:8 & $\mathrm{F}$ & $38 \dagger$ & + & NA & - & NA \\
\hline II:9 & $M$ & $40 \dagger$ & + & NA & - & $N A$ \\
\hline III:23 & M & $28 \dagger$ & + & NA & - & NA \\
\hline II:5 & M & 56 & - & FA, FLA & MP & + \\
\hline II:6 & M & $60 \dagger$ & - & FA, FLA & MP & + \\
\hline II:7 & $\mathrm{F}$ & 58 & - & FLA & MP & + \\
\hline II:10 & $M$ & 53 & - & FLA & MP & + \\
\hline III:8 & $\mathrm{F}$ & 43 & - & - & $\mathrm{CDI}$ & + \\
\hline III:15 & M & 31 & - & - & MP & - \\
\hline III:16 & $\mathrm{F}$ & 33 & - & FA & - & - \\
\hline III:18 & M & 43 & - & FLA & - & - \\
\hline III:21 & M & 39 & - & - & - & + \\
\hline III:22 & $\mathrm{F}$ & 35 & - & - & - & - \\
\hline III:24 & M & 35 & - & - & - & - \\
\hline III:27 & M & 35 & - & - & - & + \\
\hline III:28 & $\mathrm{F}$ & 20 & - & - & - & - \\
\hline
\end{tabular}

Idade em anos; + presente; - ausente; †: óbito, M: masculino; F: feminino; DCl: dispositivo cardíaco implantável; MP: marca-passo; CDI: cardioversor-desfibrilador implantável; HVE: hipertrofia ventricular esquerda; NA: não avaliado; SV: supraventricular; FA: fibrilação atrial; FLA: flutter atrial.

Em nosso estudo, a maioria dos pacientes desenvolveram hipertrofia ventricular ao longo do tempo, porém não encontramos associação desse achado com a indicação de MP. Gollob et al. descreveram complicações cardiovasculares importantes na síndrome do PRKAG2 na ausência de hipertrofia ventricular, sugerindo que essa variável não seja um fator preditor preciso.?

Outra característica marcante da síndrome do PRKAG2 é o comprometimento progressivo do sistema de condução cardíaco, com disfunção do nó sinusal e bloqueio atrioventricular. ${ }^{6}$ Em nossa coorte, o implante de MP foi frequente, acometendo 38,5\% dos pacientes. Um achado interessante foi a prevalência de flutter atrial significativamente maior nesses casos, atingindo 100\% dos maiores de 50 anos. Embora essa associação possa ser explicada meramente por coincidência de eventos, parece plausível que o flutter atrial desempenhe papel preditor de complicações cardiovasculares.

O mecanismo de morte súbita na doença é controverso; o bloqueio atrioventricular e a fibrilação ventricular 


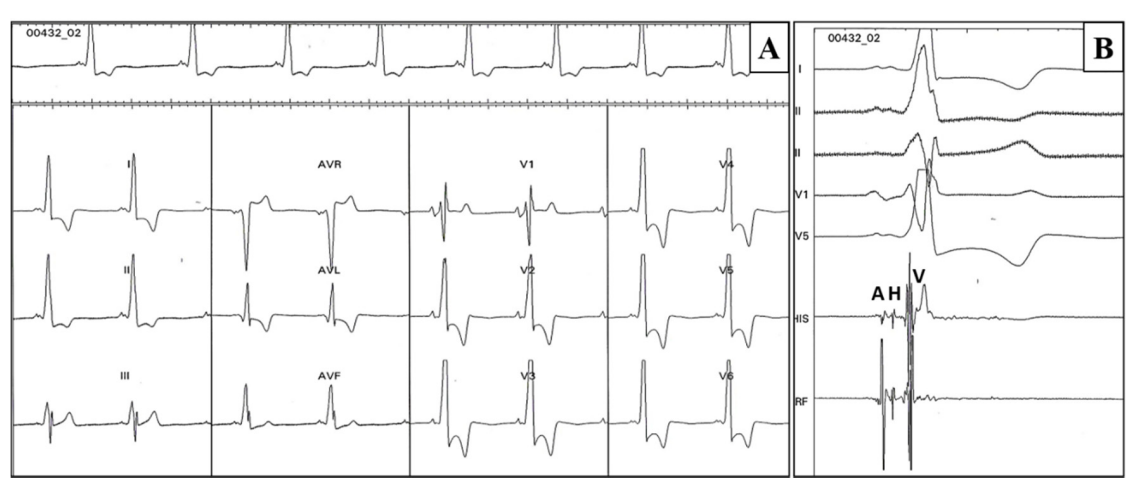

Figura 2 - A) Eletrocardiograma da paciente III:8 mostrando pré-excitação ventricular e morfologia de bloqueio de ramo direito. B) Estudo eletrofisiológico. Intervalo HV= $30 \mathrm{~ms}$. Eletrogramas, A: atrial; H: His; V: ventricular.

Tabela 2 - Análise comparativa de características clínicas entre pacientes com e sem implante de marca-passo

\begin{tabular}{lcccc}
\hline Características & Com marca-passo $\mathbf{n = 5}$ & Sem marca-passo $\mathbf{n = 8}$ & RR (IC95\%) & Valor de $\mathbf{p}$ \\
\hline Idade (anos) & $41 \pm 8,4$ & $35 \pm 7,3$ & - & 0,243 \\
\hline Idade $\geq 40$ anos (\%) & $3(60)$ & $2(25)$ & $2,4(0,6-9,7)$ & 0,293 \\
\hline Sexo masculino (\%) & $4(80)$ & $4(50)$ & $2,5(0,4-16,5)$ & 0,565 \\
\hline Hipertrofia VE (\%) & $3(60)$ & $3(38)$ & $1,8(0,4-7,2)$ & 0,592 \\
\hline Flutter atrial (\%) & $4(80)$ & $1(13)$ & $6,4(0,97-42,2)$ & 0,032 \\
\hline Síncope & $2(40)$ & $2(25)$ & $1,5(0,4-5,8)$ & 0,999 \\
\hline
\end{tabular}

VE: ventricular esquerda; RR: risco relativo; IC: intervalo de confiança.

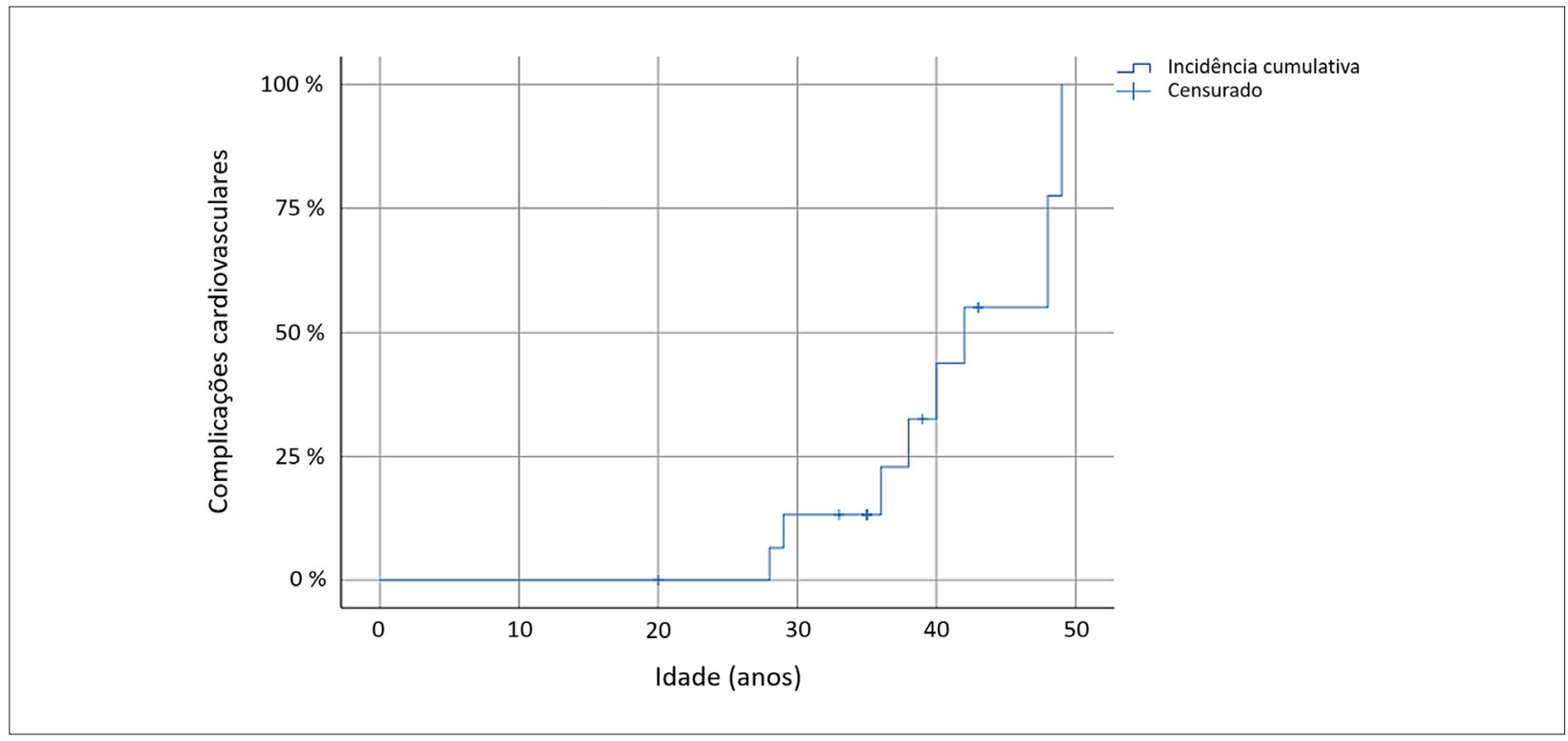

Figura 3 - Método de Kaplan-Meier: incidência cumulativa de complicações cardiovasculares (implante de marca-passo ou morte súbita). 


\section{Carta Científica}

são possíveis causas, sendo essa última provocada por fibrilação atrial com condução rápida por via acessória. ${ }^{8}$ A possibilidade de arritmia ventricular parece ser baixa, sem relatos de terapias apropriadas com cardioversor desfibrilador implantável (CDI) na literatura. Em nosso estudo identificamos via acessória fascículo-ventricular, cujo envolvimento em arritmias malignas não foi comprovado. ${ }^{9}$ Além disto, não foi registrado arritmia ventricular durante estudo eletrofisiológico ou monitorização por MP/CDI. Portanto, é provável que o bloqueio atrioventricular total tenha sido a causa de morte nesta família, potencialmente evitável com implante de MP. O desafio que persiste é identificar os pacientes com maior risco de evolução desfavorável e instituir terapia adequada preventiva.

Em conclusão, deve-se suspeitar de síndrome do PRKAG2 em pacientes jovens com pré-excitação ventricular, taquiarritmias atriais e hipertrofia ventricular familiar. A associação significativa entre aparecimento de flutter atrial e evolução para implante de marca-passo pode ter um papel no manejo dos portadores da síndrome. Futuros estudos devem esclarecer a importância clínica dessa observação.

\section{Contribuição dos autores}

Concepção e desenho da pesquisa e Análise estatística: Magalhães LP, Magalhães EFS; Obtenção de dados: Magalhães LP, Magalhães EFS, Pinheiro JO, Guabiru AT; Análise e interpretação dos dados e Redação do manuscrito: Magalhães LP, Magalhães EFS, Aras R; Revisão crítica do manuscrito quanto ao conteúdo intelectual importante: Magalhães LP, Magalhães EFS, Pinheiro JO, Guabiru AT, Reis FJFB, Aras R.

\section{Potencial conflito de interesse}

Não há conflito com o presente artigo

\section{Fontes de financiamento}

O presente estudo não teve fontes de financiamento externas.

\section{Vinculação acadêmica}

Este artigo é parte de tese de doutorado de Luiz Pereira de Magalhães pela Pós-Graduação em Medicina e Saúde da Universidade Federal da Bahia.

\section{Aprovação ética e consentimento informado}

Este estudo foi aprovado pelo Comitê de Ética do Hospital Universitário Professor Edgard Santos sob o número de protocolo 3.044.277. Todos os procedimentos envolvidos nesse estudo estão de acordo com a Declaração de Helsinki de 1975, atualizada em 2013. O consentimento informado foi obtido de todos os participantes incluídos no estudo.

\section{Referências}

1. Porto AG, Brun F, Severini GM, Losurdo P, Fabris E, Taylor MRG, et al. Clinical Spectrum of PRKAG2 Syndrome. Circ Arrhythm Electrophysiol. 2016;9(1):e003121. doi: 10.1161/CIRCEP.115.003121.

2. Zhang LP, Hui B, Gao BR. High Risk of Sudden Death Associated with a PRKAG2-Related Familial Wolff-Parkinson-White Syndrome. J Electrocardiol. 2011;44(4):483-6. doi: 10.1016/j.jelectrocard.2010.02.009.

3. Gollob MH, Green MS, Tang AS, Gollob T, Karibe A, Ali Hassan AS, et al. Identification of a Gene Responsible for Familial Wolff-Parkinson-WhiteSyndrome. NEngl J Med. 2001;344(24):1823-31.doi: 10.1056/NEJM200106143442403.

4. Arad M, Benson DW, Perez-Atayde AR, McKenna WJ, Sparks EA, Kanter RJ, et al. Constitutively Active AMP Kinase Mutations Cause Glycogen Storage Disease Mimicking Hypertrophic Cardiomyopathy. J Clin Invest. 2002;109(3):357-62. doi: 10.1172/JCl14571.

5. Sternick EB, Oliva A, Magalhães LP, Gerken LM, Hong K, Santana O, et al. Familial Pseudo-Wolff-Parkinson-White Syndrome.J Cardiovasc Electrophysiol. 2006;17(7):724-32. doi: 10.1111/j.1540-8167.2006.00485.x.

6. Thevenon J, Laurent G, Ader F, Laforêt P, Klug D, Pentiah AD, et al. High Prevalence of Arrhythmic and Myocardial Complications in Patients with Cardiac Glycogenosis Due to PRKAG2 Mutations. Europace. 2017;19(4):651-9. doi: 10.1093/europace/euw067.

7. Gollob MH, Seger JJ, Gollob TN, Tapscott T, Gonzales O, Bachinski L, et al. Novel PRKAG2 Mutation Responsible for the Genetic Syndrome of Ventricular Preexcitation and Conduction System Disease with Childhood Onset and Absence of Cardiac Hypertrophy. Circulation. 2001;104(25):3030-3. doi: 10.1161/hc5001.102111.

8. Lopez-Sainz A, Dominguez F, Lopes LR, Ochoa JP, Barriales-Villa R, Climent V, et al. Clinical Features and Natural History of PRKAG2 Variant Cardiac Glycogenosis. J Am Coll Cardiol. 2020;76(2):186-97. doi: 10.1016/j. jacc.2020.05.029.

9. Rohrhoff NJ, Finne HA, Rodriguez Y. A Sailor's Dilemma: A Case of Preexcitation via a Fasciculoventricular Pathway. HeartRhythm Case Rep. 2017;3(7):364-7. doi: 10.1016/j.hrcr.2017.05.013. 